This item was submitted to Loughborough's Research Repository by the author.

Items in Figshare are protected by copyright, with all rights reserved, unless otherwise indicated.

\title{
Effects of table design in railway carriages on pregnant occupant safety
}

PLEASE CITE THE PUBLISHED VERSION

http://dx.doi.org/10.1080/13588265.2012.664009

\section{PUBLISHER}

(c) Taylor \& Francis

\section{VERSION}

AM (Accepted Manuscript)

\section{PUBLISHER STATEMENT}

This work is made available according to the conditions of the Creative Commons Attribution-NonCommercialNoDerivatives 4.0 International (CC BY-NC-ND 4.0) licence. Full details of this licence are available at: https://creativecommons.org/licenses/by-nc-nd/4.0/

\section{LICENCE}

CC BY-NC-ND 4.0

\section{REPOSITORY RECORD}

Esat, Volkan, and B. Serpil Acar. 2019. "Effects of Table Design in Railway Carriages on Pregnant Occupant Safety". figshare. https://hdl.handle.net/2134/23436. 


\title{
Effects of table design in railway carriages on pregnant occupant safety
}

\author{
V. Esat ${ }^{\mathrm{a}}$ and B. Serpil Acar ${ }^{\mathrm{b} *}$ \\ ${ }^{a}$ Mechanical Engineering Program, Middle East Technical University - Northern Cyprus Campus, Guzelyurt, Mersin 10, Turkey; \\ ${ }^{b}$ Design School, Loughborough University, Loughborough, LE11 3TU, UK
}

\begin{abstract}
This paper focuses on safety investigations for pregnant occupants, in particular, on their interactions with an interior feature, fixed bay tables, in railway vehicles. The computational pregnant occupant model Expecting has represented pregnant travelers in railway vehicle environments. Expecting is a computational pregnant occupant model developed at Loughborough University, in order to investigate the dynamic response of pregnant women to impacts. It has been successfully utilised by the authors in earlier studies, in various automobile crash investigations, such as frontal impacts with real and simplified crash pulses. In this study, a model of a network train carriage is generated and used together with Expecting to assess the suitability of fixed bay table designs for pregnant occupants. Investigations of potential injuries in this paper are believed to contribute to the design of more suitable interior features and hence improve safety and quality of life for pregnant women as travelers in railway vehicles.
\end{abstract}

Keywords: pregnant traveler; placental abruption; railway vehicle; network train; interior design; fixed bay table

\section{Introduction}

In the United Kingdom, on average 2.75 million people take trains every weekday, and approximately 4.25 million passengers take the London Underground daily. It is also reported that total number of passenger journeys in the United Kingdom in 2008-2009 for franchised operated services increased by $4.0 \%$ from 1.22 billion in 2007-2008 to 1.27 billion in 2008-2009 [4]. The total passenger revenue for franchised operated services increased to $£ 6.0$ billion in 2008-2009 showing an 8.1\% increase from 2007-2008 figures. The increase in revenue from 20062007 to $2007-2008$ is $10.8 \%$ [4]. These figures show the potential and justify the need to invest more in public transport.

No passenger or workforce fatalities were reported in 2009 in train accidents in the United Kingdom. A few fatalities were recorded due to accidents happened in stations [6]. In 2008, 70 accidental (such as level crossing incidents) fatalities, 422 major injuries and 12,308 incidents of minor injuries or shock/trauma were recorded [5]. It is also presented in the same report that the proportions of male and female travelers are quite close, with female travelers constituting $46 \%$ of all journeys. Over the five-year period 2004-2008, it is claimed that females were over-represented in overall levels of harm, although male fatality rates were higher, which implies that females were involved in a higher number of lower severity accidents than males [5].

The primary collision in railway vehicles represents the initial stage, in which the carriages collide and the initial kinetic energy is dissipated through plastic structural deformation. Crash pulses occurring as a result of this stage are reflected onto the internal contents of the railway vehicle, both onto the physical interior environment and the occupants. The secondary collision is the second stage where the occupant collides with the interior elements and/or other occupants. Therefore, the dynamic response of the occupants needs to be investigated in terms of injury mechanisms and criteria in relation with the crash pulse acting on them.

In railway passenger carriages, various seat configurations are used. Mainly the seats are named according to the direction of travel, such as forward and backing. Some of the seats are called airline seats as they are configured similar to airline seats (have a small fold down table attached to the seat in front and retractable arm rests) and some are called seats around a fixed bay table. Seats close to the carriage doors, usually with ample leg space, are called priority seats. This research is concerned with the configuration of seats around a fixed bay table.

No detailed information is available on injuries of pregnant women in railway vehicle incidents. This research aims to investigate the effects of one of the interior features of railway vehicles, fixed bay tables, on pregnant women during low velocity impacts $(\Delta V)$ and sudden deceleration situations. The impact of railway vehicle interior designs on pregnant traveler safety is investigated by using a computational model of a network train carriage together with Expecting. 


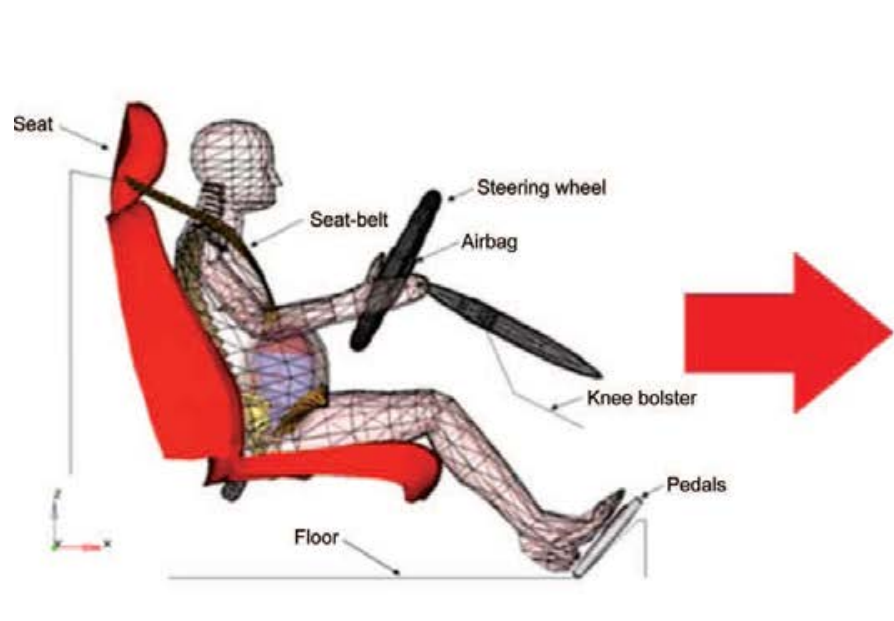

(a)

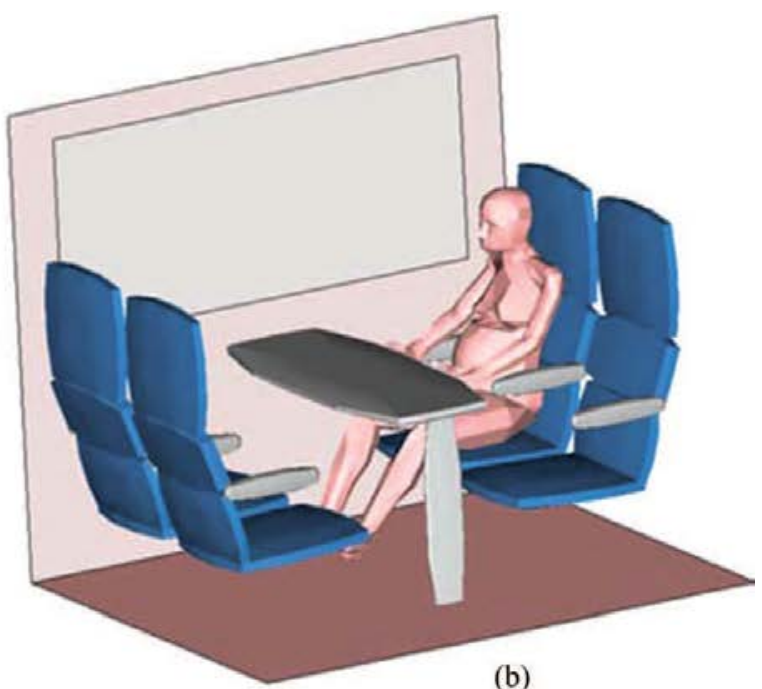

(b)

Figure 1. Expecting in (a) automobile medium, and (b) a network train environment.

The three-dimensional computational model Expecting, which includes a detailed multi-body model of a 38week-old fetus in a finite element (FE) model of a uterus with a placenta, was developed by Acar and van Lopik at Loughborough University, United Kingdom [1]. Expecting was initially developed for automobile environments; however, in this project the computational pregnant occupant model is placed in a railway vehicle medium.

\section{Methodology}

The computational pregnant occupant model Expecting is used to investigate the dynamic responses of the pregnant occupant and the fetus within a network train model during a low and a relatively higher velocity sudden deceleration cases.

\section{Computational modelling: Expecting in a network train}

The computational pregnant women model Expecting was modelled to reflect various anthropometric and anatomical changes of pregnancy as shown in Figure 1. Expecting is a female occupant model representing a 5 th percentile female in her 38th week of pregnancy. The model embodies a detailed multi-body representation of a fetus within a FE uterus as shown in Figure 2. Expecting was constructed in the MADYMO software of TNO Automotive [3]. Details of the development and validation of Expecting are published by Acar and van Lopik in [1].

In this research project, Expecting is taken from the automobile medium and placed into a network train carriage model as seen in Figure 1. The technical details of the train interiors are provided by Bombardier Inc. [7].

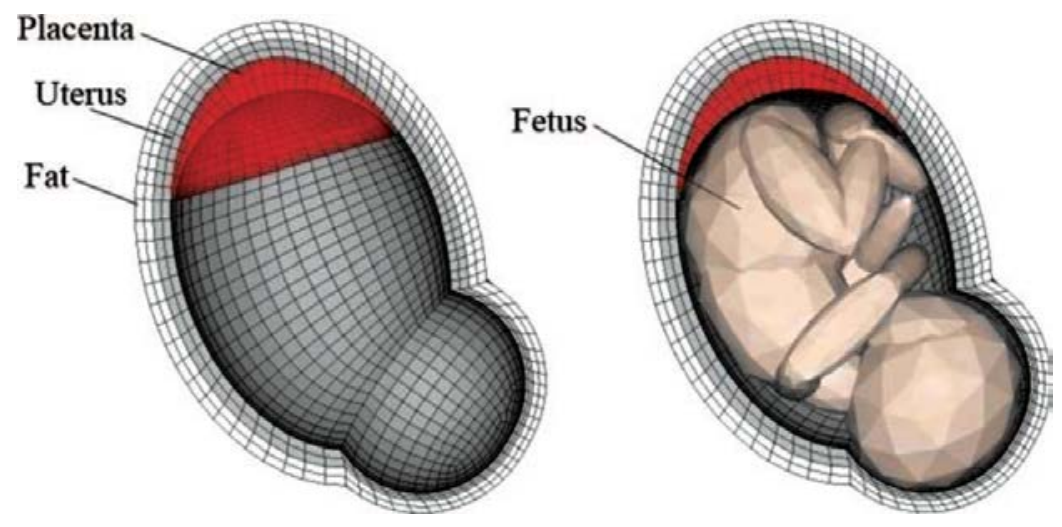

Figure 2. The uterus, placenta and fetus in Expecting. 


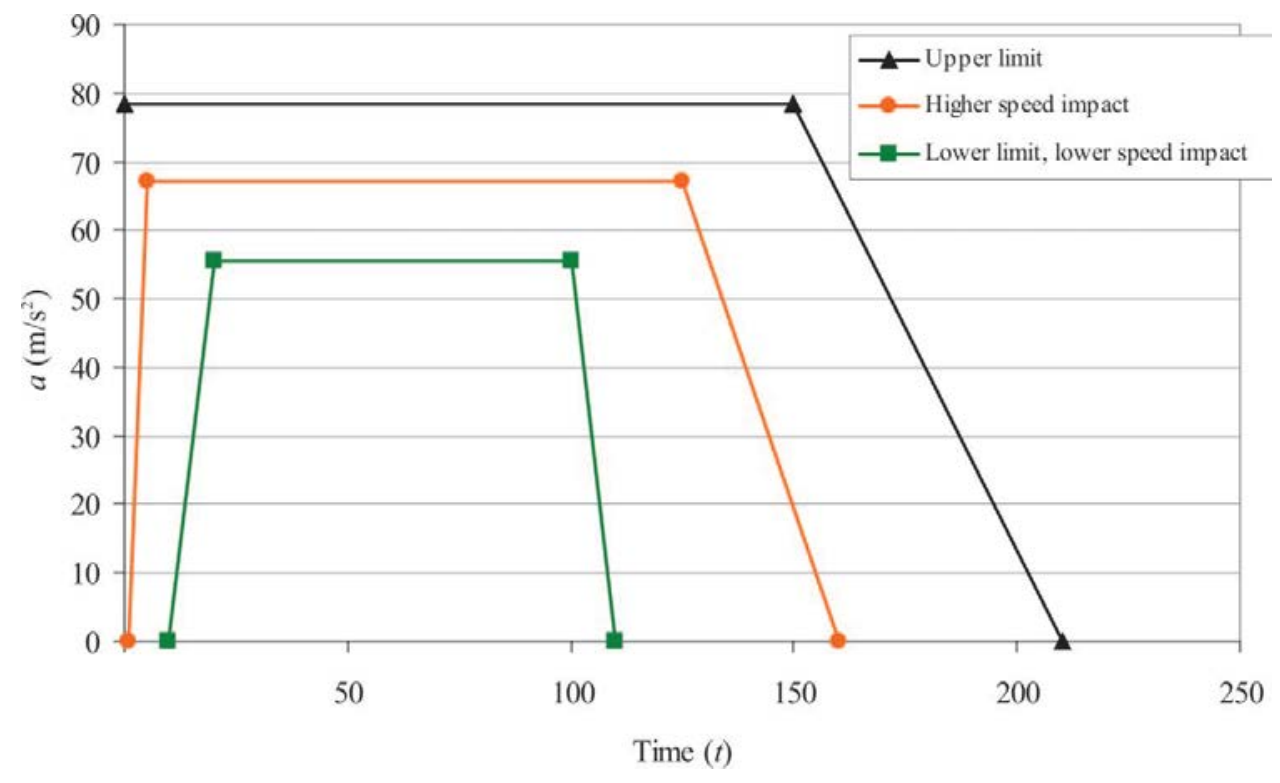

Figure 3. Input deceleration crash pulses.

Seats around the fixed bay tables are usually not identical and the distance between the seats and the table can vary according to the location of the seat group. The safety of pregnant travelers is investigated for a range of distances between the pregnant abdomen and the floor-mounted table, as well as of the table height and thickness in search of the most suitable seating group.

The seats are modelled by using realistic dimensions and orientations by employing super ellipsoid surfaces. The segments of the seats are then combined together in order to form the whole seating configuration. Four seats and the floor-mounted bay table are positioned by using dimensions from the network train carriage drawings [7], as well as the sidewall and the window. All surfaces are associated with necessary contact model definitions within MADYMO to facilitate a realistic medium as the secondary impact occurs between the pregnant women and the fixed bay table.

The FE uterus model was developed in line with the multi-body fetus model with the fetal dimensions and configuration determining the dimensions of the uterus by giving a snug fit around the fetus as shown in Figure 2. A layer of fat is also meshed around the outer surface of the uterus. The resulting total mass of the uterus with placenta is $1.29 \mathrm{~kg}$ and the mass of the fetus is $3.3 \mathrm{~kg}$. The pregnant occupant model is validated against rigid-bar impact and belt-loading tests [1].

\section{Simulation parameters}

Simulations are carried out with lower velocity and higher velocity deceleration pulses representing sudden braking or impact. The floor-mounted bay table is selected as the main train interior feature in order to investigate the effects of varying distance between the table and the pregnant abdomen (TD), and table height (TH) and table thickness (TTh) on the safety of the pregnant traveler. In the lower $\Delta V$ simulations, the pulse corresponds to the lower boundary of the recommended pulse corridor in the AV/ST9001 standard [2] with an impact velocity $(\Delta V)=$ $18 \mathrm{kph}$ and an impact duration $(\Delta t)=100 \mathrm{~ms}$. In the higher $\Delta V$ simulations, the chosen trapezoidal crash pulse represents a reaively higher velocity sudden deceleration condition with $\Delta V$ around $34 \mathrm{kph}$ and $\Delta t=160 \mathrm{~ms}$. The pulses are illustrated in Figure 3.

In the model, the floor and the table are considered to be rigid. Seats are modelled as a collection of rigid bodies; however, friction between the seat pan and the occupant is incorporated with an average kinetic friction coefficient

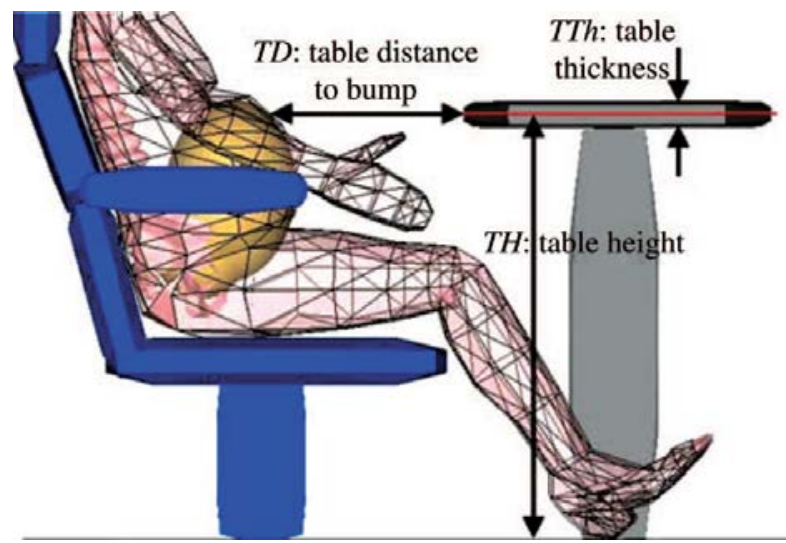

Figure 4. Network train interior design parameters that are varied for the simulations. 
Lower speed impact

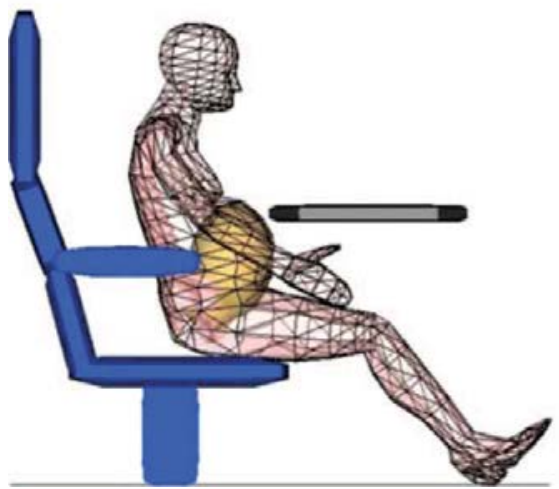

Higher speed impact

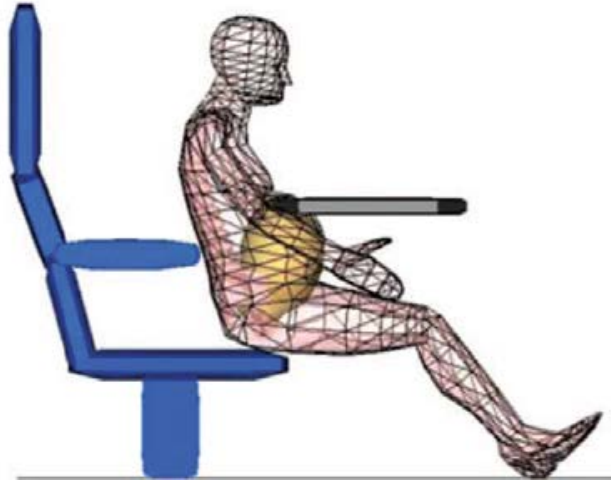

Figure 5. Views from lower velocity impact $(\Delta V=18 \mathrm{kph})$ and higher velocity impact $(\Delta V=34 \mathrm{kph})$ simulation cases for $T H=62 \mathrm{~cm}, T D=20 \mathrm{~cm}$, and $T T h=4 \mathrm{~cm}$ at $100 \mathrm{~ms}$.

of 0.5 [8]. MADYMO is programmed to check contactbetween all contacting elements of the model, namely between the occupant, and the seats and the table.

In both sets of simulations, three design parameters are considered. The table height $(\mathrm{TH})$ is defined as the vertical distance from the central plane of the table to the floor. The other variables constitute the horizontal distance from the closest point of the table (on the central plane) to the bump (TD) and the thickness of the table (TTh). These parameters are shown in Figure 4. In all simulations, the seat height, vertical distance between the seat pan and the floor is kept constant as in the technical drawings. $T D$ value is varied between $4 \mathrm{~cm}$ and $27 \mathrm{~cm}$, while $T H$ and $T T h$ are kept constant at $62 \mathrm{~cm}$ and $4 \mathrm{~cm}$, respectively. $T H=62 \mathrm{~cm}$ is the standard table height taken from technical drawings. Again, for each set of simulations, $T H \quad$ is varied between $56 \mathrm{~cm}$ and $62 \mathrm{~cm}$, while the other variables are kept constant. These simulations are carried out for TDs $20 \mathrm{~cm}$ and $24 \mathrm{~cm}$ with TTh of $4 \mathrm{~cm}$. Finally, $T T h$ is varied from 2 to $6 \mathrm{~cm}$ for the $T H$ value of 62 cm with constant $T D=20 \mathrm{~cm}$. The reason behind choosing specific $T H$ and $T D$ values while varying $T T h$ is discussed in the next section.

\section{Injury criterion}

In this study, only the maximum von Mises equivalent strain levels in uterus at placental location, utero-placental interface (UPI), are collected to assess the possibility of placental abruption, which results in fetal, and occasionally maternal death. The threshold strain value for the occurrence of placental abruption is widely accepted to be 0.60 at the UPI [9].

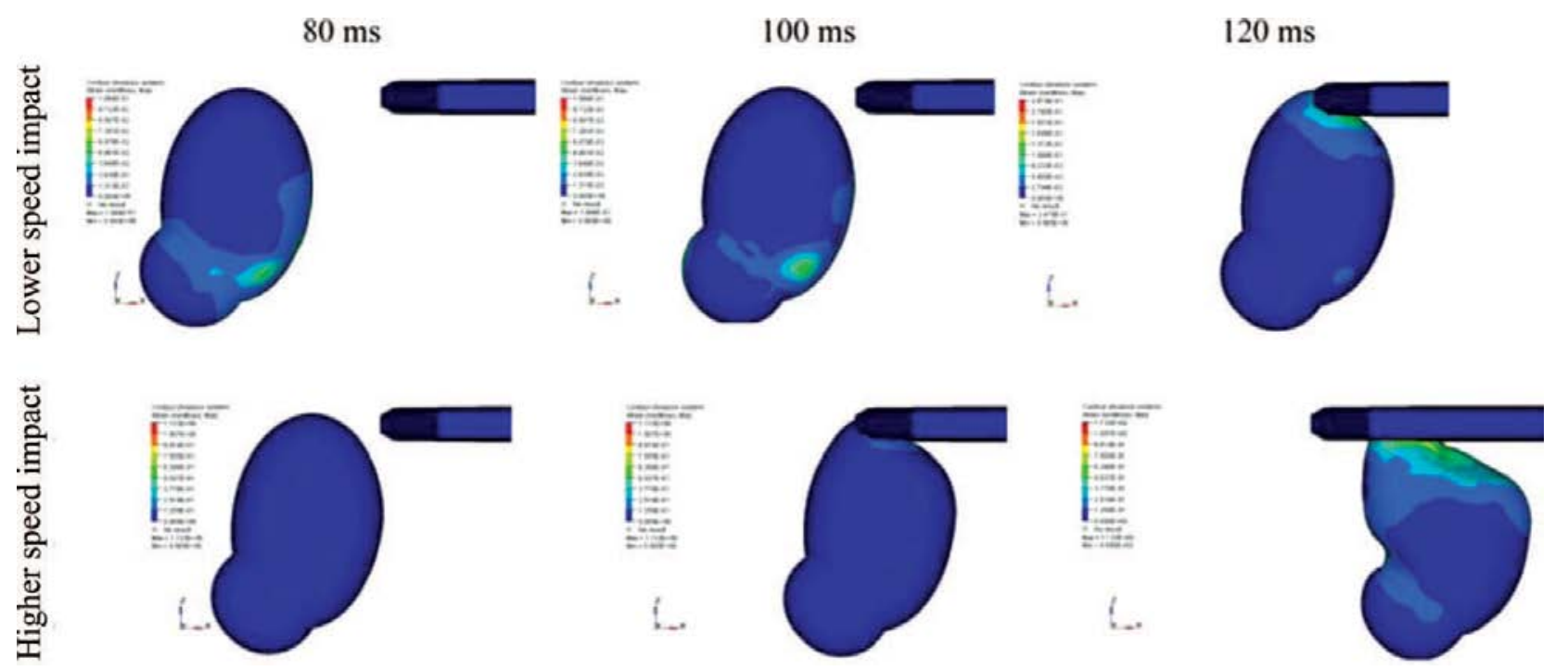

Figure 6. Uterus deformations from lower velocity impact $(\Delta V=18 \mathrm{kph})$ and higher velocity impact $(\Delta V=34 \mathrm{kph})$ simulation cases for $T H=62 \mathrm{~cm}, T D=20 \mathrm{~cm}$, and $T T h=4 \mathrm{~cm}$. 


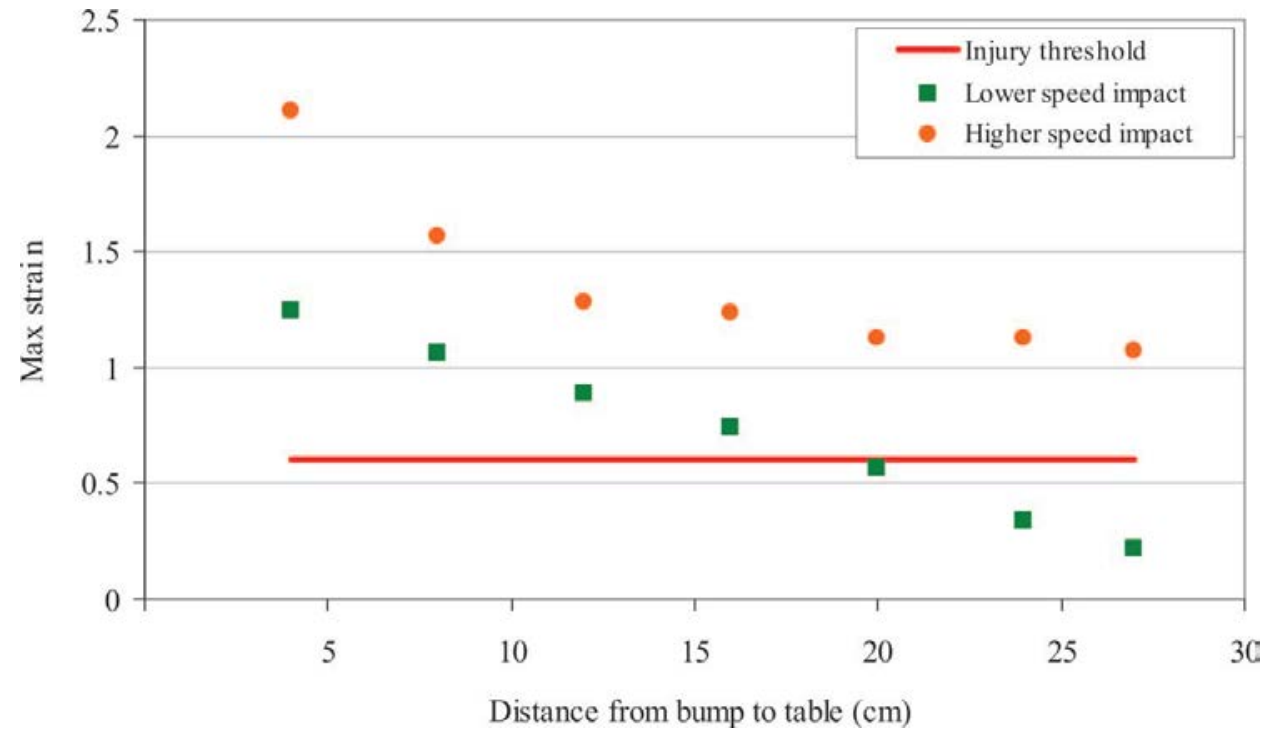

Figure 7. Maximum strains at the UPI with varying $T D$.

\section{Results and discussion}

Maximum von Mises equivalent strains in the uterus at the UPI are found to be the highest strains in the overall uterus. Therefore, all strain values presented are associated with the UPI region, which can directly be related to placental abruption risk. Typical simulation outputs for the two impact severities at $100 \mathrm{~ms}$ and the strain distribution in the uterus for the two impact severities at 80, 100 and $120 \mathrm{~ms}$ are given in Figures 5 and 6, respectively. In both figures, $\Delta V=18 \mathrm{kph}$ and $34 \mathrm{kph}$ simulations represent the case with $T H=62 \mathrm{~cm}, T D=20 \mathrm{~cm}$ and $T T h=4 \mathrm{~cm}$. It is shown in Figure 6 that in general the maximum strains occur at the UPI region for both impact conditions. Higher $\Delta V$ causes the pregnant traveler to displace more severely forwards and below the table, showing a 'submarining' pattern.

The three sub-cases of varying parameter values are presented separately, where each parameter is compared and analysed for the two impact severities, lower velocity impact $(\triangle V=18 \mathrm{kph})$ and higher velocity impact $(\Delta V=34$ kph).

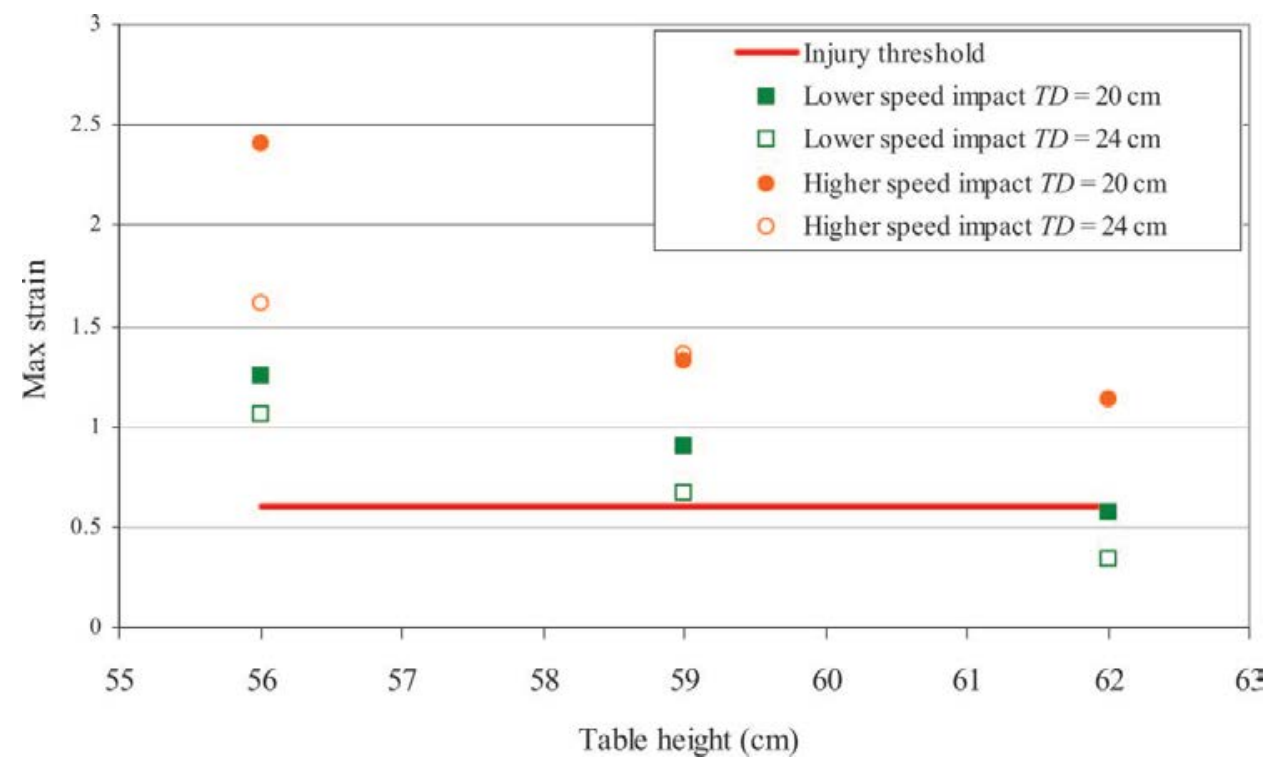

Figure 8. Maximum strains at the UPI with varying $T H$. 


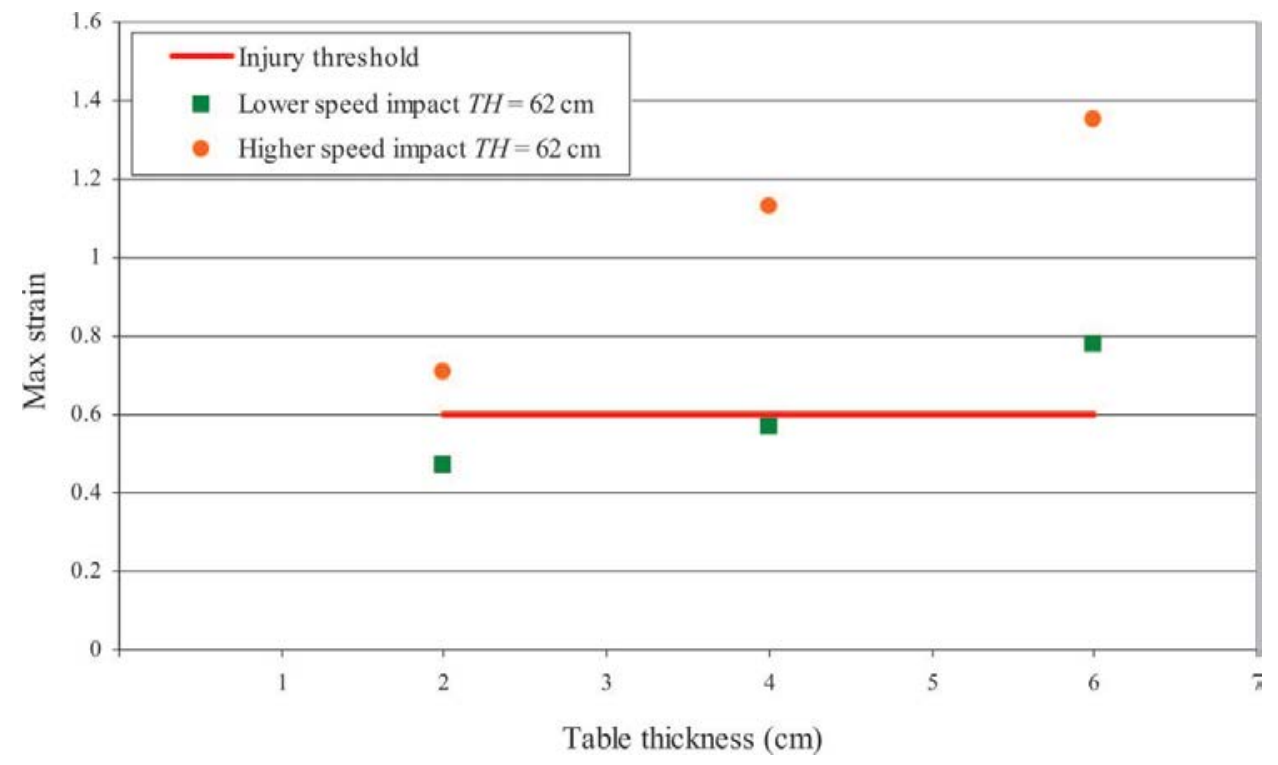

Figure 9. Maximum strains at the UPI with varying TTh.

\section{Varying the horizontal distance between the table and the pregnant abdomen (TD)}

In order to investigate the effect of the distance between the table and the 'bump' on pregnant traveler safety, $T D$ is varied from 4 to $27 \mathrm{~cm}$ while $T H$ and TTh are kept constant at $62 \mathrm{~cm}$ and $4 \mathrm{~cm}$, respectively. Variations in $T D$ represent a possible shorter seat-to-table distance seating configuration or pregnant woman sitting somehow closer to the table. The strain results are provided in Figure 7.

Figure 7 shows that even at a lower severity impact, with a distance less than $20 \mathrm{~cm}$ between the table and the bump, placental abruption is highly probable. With the higher severity impact, for every distance between the table and the bump, placental abruption is almost certain.

It is worth noting that a railway occupant might be expected to be at higher risk when the distance between the occupant and the bay table is larger, since the relative velocity between the occupant and the bay table increases as the railway vehicle decelerates. However, this study reveals that when the gap between the pregnant abdomen and the table (TD) is larger, a direct impact to the placenta by the bay table is mostly avoided as the pregnant occupant 'submarines' under the baytable.

$$
T T h=2 \mathrm{~cm}
$$
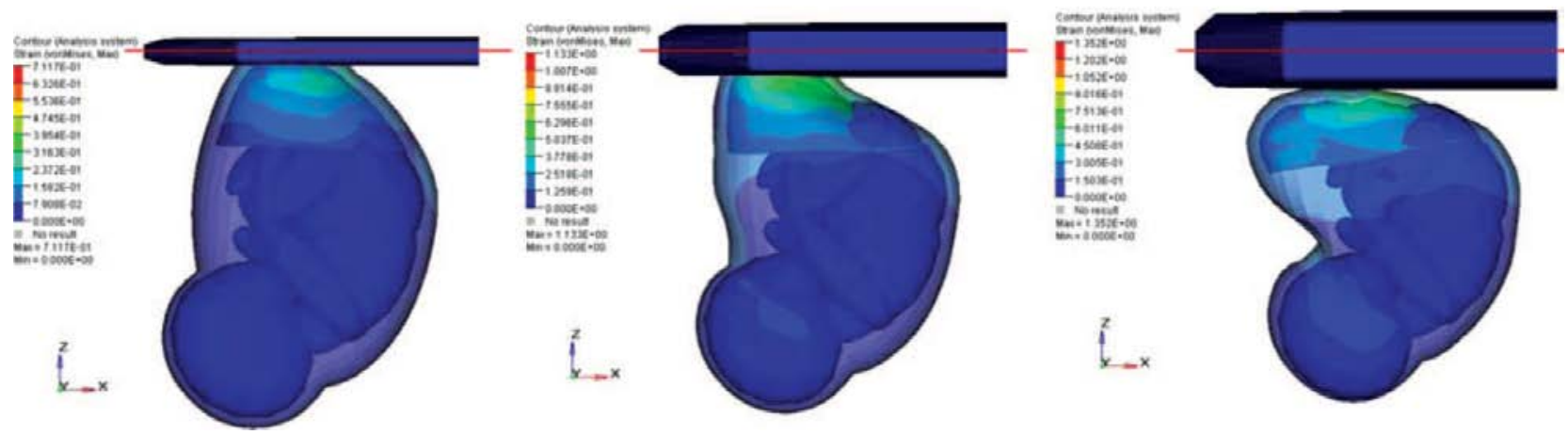

Figure 10. Strain distribution in uterus with varying TTh with views of fetus and placenta through the transparent uterus for higher velocity impact, $\Delta V=34 \mathrm{kph}$. 


\section{Varying the table height (TH)}

In order to assess the effects of $T H$ on safety, $T H$ is varied from a height of $62 \mathrm{~cm}$ to a lower height of $56 \mathrm{~cm}$ while the other variables are kept constant. These simulations are carried out for $T D=20$ and $24 \mathrm{~cm}$ with $T T h=4 \mathrm{~cm}$. As $T D=20 \mathrm{~cm}$ is found as the critical lower limit for design in the previous section, only TDs over $20 \mathrm{~cm}$ are compared in this section. The results are depicted in Figure 8.

The strain values at the UPI for variations in $T H$ present a highly risky condition for the pregnant traveler. Only for the highest setting of $62 \mathrm{~cm}$ and only for lower velocity impact $(\triangle V=18 \mathrm{kph})$ with $T D=20$ and $24 \mathrm{~cm}$, the strain value is below the threshold. For all other $T H$ values, for both the severities and each proximity setting ( $T D=20$ and $24 \mathrm{~cm}$ ), placental abruption may potentially happen. However, the figure also shows a downward trend for all cases as the $T H$ value increases.

\section{Varying the table thickness (TTh)}

The variation in TTh from 2 to $6 \mathrm{~cm}$ for $T H$ value of $62 \mathrm{~cm}$ and with a constant $T D$ of $20 \mathrm{~cm}$ is investigated. The results are presented in Figure 9. The graph shows that for the lower $\Delta V$, with $T H=62 \mathrm{~cm}$, table thickness can play a significant role in placental abruption risk. For example, TTh at $4 \mathrm{~cm}$ gives just below the threshold values for placental abruption whereas for TTh over $6 \mathrm{~cm}$, placental abruption is highly probable. For the higher $\Delta V$, all $T T h$ values show placental abruption risk and the risk of placental abruption increases with increasing TTh values, as shown in Figure 9. The results show that lower TTh values provide smaller risk in terms of placental abruption; however, reducing TTh, in general, may increase injury severity due to increased thoracic intrusion.

For the higher $\Delta V$, with $T D=20 \mathrm{~cm}$ and $T H=62 \mathrm{~cm}$ case, the strain distributions and the effect of the increasing

TTh on uterus deformation is clearly illustrated for 120-ms frame in Figure 10.

\section{Conclusions}

The assessments and analyses carried out in this study reveal that interior features of railway vehicles may potentially affect safety of pregnant women. This research suggests that placental abruption risk increases by close proximity, low and thick tables. Considering standard UK railway vehicle designs, for the floor-mounted table seating group, the optimum settings for pregnant women appear to be a $\mathrm{TH}$ of at least $62 \mathrm{~cm}$ - from the central plane of the table to the floor - and a TTh, such as $2 \mathrm{~cm}$.

The research also suggests that the seats that allow as much space between the pregnant abdomen and the table, at least $20 \mathrm{~cm}$, are sensible for pregnant women to occupy for minimum placental abruption risks at lower $\Delta V$ (such as $\Delta V=18 \mathrm{kph}$ ).
It is of course important to consider the well-being of the mother-to-be as well as the fetus, and the interiors of the trains must not be designed for mutually exclusive benefits. Therefore, when considering changing the dimensions of the internal design features, for example, reducing the table thickness, injury risks to pregnant women herself and to the fetus should be considered simultaneously.

The authors acknowledge the fact that there are many constraints in railway vehicle designs, mostly shaped by financial and technical issues and ergonomic considerations for all passengers. There could be many variations among women and between pregnancies of the same woman. However, the computational tool Expecting can be used in fine- tuning the designs, to predict optimal settings for minimum placental abruption risks and to generate specifically designed priority seats for pregnant women.

The findings of this paper warrant further research to investigate other interior design features, and under different impact conditions.

\section{Acknowledgements}

The authors thank the EPSRC and the Innovative Manufacturing and Construction Research Centre (IMCRC) of Loughborough University for sponsoring 'Rail Transport Safety for Pregnant Occupants Preliminary Investigations’ research project, Bombardier Transportation UK Ltd for useful technical information and MADYMO. The authors also thank the referees for their useful comments that helped improve the paper.

\section{References}

[1] B.S. Acar and D. van Lopik, Computational pregnant occupant model, 'Expecting', for crash simulations, Proc. IMechE Part D, J. Automob. Eng. 223 (2009), pp. 891-902.

[2] ATOC Vehicles Standard, AV/ST9001, Vehicle interior crashworthiness, UK ATOC Stand. 1 (Feb 2002), 1-27.

[3] MADYMO, TNO MADYMO BV v7.0, in MADYMO Theory Manual, TASS, Livonia, MI, 2007.

[4] Office of Rail Regulation (ORR), National Rail Trends, Chapter 1: Rail Usage, GB, ORR, London, 2009, p. 15.

[5] Rail Safety and Standards Board, Annual Safety Performance Report 2008, GB, Rail Safety and Standards Board Limited, London, 2008, p. 290.

[6] Rail Safety and Standards Board, Overview of Safety Performance for 2009, GB, Rail Safety and Standards Board Limited, London, 2009, p. 4.

[7] Rail Safety and Standards Board, Technical Drawings for DMOS Railway Vehicle for LERL Stansted Express, Bombardier, Belfast, UK, 2009.

[8] J. Rasmussen, S. Tørholm, and M. de Zee, Computational analysis of the influence of seat pan inclination and friction on muscle activity and spinal joint forces, Int. J. Ind. Ergonom. 39 (2009), pp. 52-57.

[9] J.D. Rupp, K.D. Klinich, S. Moss, L. Zhou, M.D. Pearlman, and L.W. Schneider, Development and testing of a prototype pregnant abdomen for the small female Hybrid III small female ATD, Stapp Car Crash J. 45 (2001), pp. 61-78. 